\title{
What is the Impact of the European Resuscitation Council Covid-19 Guidelines on Resuscitation Quality - A Manakin Study
}

Gerrit Jansen ( $\square$ gerritjansen@web.de )

Protestant Hospital Bethel: Evangelisches Klinikum Bethel gGmbH

Nicole Ebeling

Protestant Hospital Bethel: Evangelisches Klinikum Bethel gGmbH

Eugen Latka

Study Institute Westfalen-Lippe

Stefan Krüger

Protestant Hospital Bethel: Evangelisches Klinikum Bethel gGmbH

Sean Scholz

Protestant Hospital Bielefeld gGmbH: Evangelisches Klinikum Bethel gGmbH

Simon Trapp

Protestant Hospital Bethel: Evangelisches Klinikum Bethel gGmbH

Julia Johanna Grannemann

Bielefeld Municipal Hospitals: Klinikum Bielefeld gemGmbH

Daniel Thaemel

Study Institute Westfalen-Lippe

Suraj Chandwani

Protestant Hospital Bethel: Evangelisches Klinikum Bethel gGmbH

Odile Sauzet

University of Bielefeld: Universitat Bielefeld

\section{Sebastian Rehberg}

Protestant Hospital Bethel: Evangelisches Klinikum Bethel gGmbH

Rainer Borgstedt

Protestant Hospital Bethel: Evangelisches Klinikum Bethel gGmbH

\section{Research}

Keywords: advanced life support, cardiopulmonary resuscitation, Covid-19, ERC guidelines, orotracheal intubation; out-of-hospital CPR, Sars-CoV-2

Posted Date: October 1st, 2020

DOI: https://doi.org/10.21203/rs.3.rs-77425/v1

License: (c) (i) This work is licensed under a Creative Commons Attribution 4.0 International License. Read Full License 


\section{Abstract}

\section{Background}

To evaluate the effects of European Resuscitation Council (ERC) Covid-19-guidelines on resuscitation quality.

\section{Methods}

In an observational manikin study paramedics and emergency physicians performed Advanced-Cardiac-Life-Support in three settings: ERC guidelines 2015 (Control), Covid-19-guidelines as suggested with minimum staff (Covid-19-minimal-personnel); Covid-19-guidelines with paramedics and an emergency physician (Covid-19-advanced-airway-manager). Main outcome measures were no-flow-time, quality metrics as defined by ERC and time intervals to first chest compression, oxygen supply, intubation and first rhythm analysis. Data were presented as mean (standard deviation).

\section{Results}

30 resuscitation scenarios were completed. No-flow-time was markly prolonged in Covid-19-minimal-personnel [113 \pm 37 sec] compared to Control [ $55 \pm 9$ sec; $p<0.001$ ] and Covid-19-advanced-airway-manager [76 \pm 38 sec; $p<0.001$ ]. In both Covid-19-groups chest compressions started later [Control: $21 \pm 5$ sec, Covid19-minimal-personnel: $32 \pm 6$ sec; Covid-19-advanced-airway-manager: $37 \pm 7$ sec; each $p<0.001$ vs. control], but oxygen supply [Control: $77 \pm 19$ sec; Covid-19minimal-personnel: $29 \pm 5$ sec; Covid-19-advanced-airway-manager: $34 \pm 7$ sec; each $p<0.001$ vs. control] and first intubation attempt [Control: $178 \pm 44$ sec; Covid-19-minimal-personnel: $111 \pm 14 \mathrm{sec}$; Covid-19-advanced-airway-manager: $131 \pm 20 \mathrm{sec}$; each $\mathrm{p}<0.001$ vs. control] were earlier than in the control group. However, succesful intubation was similar [Control: $198 \pm 48$ sec; Covid-19-minimal-personnel: $181 \pm 42$ sec; Covid-19-advanced-airway-manager: $130 \pm 25$ sec] due to a longer intubation time in Covid-19-minimal-personnel [61 $\pm 35 \mathrm{sec}]$ compared to Covid-19-advanced-airway-manager ( $p=0.002)$ and control [19 \pm 6 sec; $p<0.001]$. Time to first rhythm analysis was more than doubled in Covid-19-minimal-personnel [138 \pm 96 sec] compared to control [50 \pm 12 sec, $p<0.001]$.

\section{Conclusion}

Covid-19-guidelines led to earlier attempts at intubation, delay in starting chest compressions, longer interruption in chest compression and markedly worsen the quality of resuscitation. These negative effects are attenuated by increasing the number of staff and addition of an experienced airway manager. Specific indications for Covid-19-guidelines are urgently required to carefully balance the risk of infection with SARS-CoV-2 for the staff vs. the potentially worse outcome for the patients.

\section{Introduction}

The rapid spread of the Covid-19 pandemic around the world poses particular challenges, even for highly developed health systems ${ }^{1}:$ In addition to managing the pandemic, maintaining a high quality of medical care for the general population is of fundamental importance ${ }^{2}$.

Before the Covid-19 pandemic guidelines of the European Resuscitation Council (ERC) 2015 provided the best evidence, for carrying out high-quality cardiopulmonary resuscitation in Europe. Based on current evidence the main focus was applied to minimizing no-flow-time by early and correct chest compressions as well as an early rhythm analysis and defibrillation ${ }^{3}$.

Currently, there is no valid evidence on the risk of SARS-CoV-2 infection for resuscitation providers ${ }^{4-5}$. However, data from severe acute respiratory syndrome in 2003 and our existing knowledge on the transmission of SARS-CoV-2 indicate that aerosol generating measures during resuscitation such as chest compressions, mouth-to-mouth- and bag-mask-ventilation may be associated with a relevant risk of infection and subsequent morbidity and mortality for medical staff performing resuscitation ${ }^{6-8}$. Prioritising rescuer safety given the uncertainty of risk of COVID-19 infection to rescuers, ERC guidelines were adapted during the Covid-19 pandemic with the primary focus on the best possible protection of medical staff, protection of colleagues and bystanders, acknowledging that there might be delays in some interventions ${ }^{9}$. Table 1 summarizes the main differences in the recommendations for resuscitation between Covid-19 guidelines and ERC recommendations $2015^{4-5,9}$.

Table 1: Major changes between Covid-19 resuscitation guidelines and ERC recommendations $2015^{3,4,9}$ 


\section{ERC 2015-guidelines}

Check the victim for a response

Open the airway

Look, listen and feel for normal breathing

\section{COVID-19-guidelines}

Check for signs of life/pulse

Do not listen for breaths or place your cheek near to the patient's face

- Place a cloth/towel over the person's mouth and nose before performing chest compressions

\section{Start chest compressions} of $30: 2)$

Combine chest compressions with rescue breaths (in a ratio

Minimise interruptions and ensure high-quality compressions

Rapid assessment of initial rhythm and early defibrillation

Maintain the airway and ventilate the lungs with the most appropriate equipment (Pocket mask ventilation, two-rescuer bagmask ventilation, supraglottic airway device)

Tracheal intubation should be attempted only by those who are trained, competent and experienced in this skill

Minimal protective equipment (gloves, work clothes)
Do not proceed with chest compressions or airway interventions without airborne-precaution PPE

Rapid assessment of initial rhythm and early defibrillation
BLS teams less skilled or uncomfortable with bag-mask ventilation should not provide bag-mask ventilation. They should place an oxygen mask on the patient's face, give oxygen and provide compression-only CPR

Experienced airway staff should insert a supraglottic airway or intubate the trachea early so that the period of bag-mask-ventilation is minimised its use

Consider videolaryngoscopy for tracheal intubation by providers familiar with

The minimum airborne-precaution PPE comprises:

Gloves

Long-sleeved gown

Filtering facepiece (FFP2/3)

Eye and face protection (e.g. full-face shield)

Viral filter [HME or HEPA]

Restrict the number of staff

Allocate a gatekeeper

All personnel not immediately needed should keep their distance from the patient and remain protected

Legend: ERC=European Resuscitation Council; Covid-19=Corona-Virus-Disease 2019; PPE= personal protective equipment; BLS= Basic-Life-Support; $\mathrm{CPR}=$ Cardio-pulmonary Resuscitation; HME=heat and moisture exchanger filter; HEPA=High Efficiency Particulate Air filter

However, due to the rapid progress of the present pandemic, these recommendations were published without evidence-based knowledge on their impact on quality of resuscitation and the subsequent potential effects on outcome of the patients ${ }^{9}$. Furthermore, a valid evaluation based on data from registries will be possible only in the remote future. Nevertheless, this information is necessary to optimise the recommendations and prevent or minimise a deterioration of the outcome.

Against this background, the present simulated manikin study evaluates the effects of the Covid-19 ERC guidelines on established quality indicators of cardiopulmonary resuscitation as well as circulatory and respiratory interventions in comparison to the previous recommendations of the ERC 2015 and provides the first evidence available 1,3-4,9. $^{1}$

\section{Methods}

Ethical approval for this study was provided by the Ethical Committee of the University Hospital of Muenster, Muenster, Germany (Ethical Committee No 2020-504-f-S). The study was performed at the 'Studieninstitut fuer kommunale Verwaltung Westfalen-Lippe, Fachbereich Medizin und Rettungswesen', Bielefeld, Germany. After providing written informed consent, 10 teams of professional paramedics and emergency physicians trained in Advanced-CardiacLife-Support (ACLS) were randomly selected and asked to perform resuscitation according to the guidelines of the ERC 2015 with the crew of an ambulance (two paramedics) and one additional paramedic and an emergency physician experienced in advanced airway management according to the emergency physician-based rescue service system in Germany (control group) ${ }^{3}$. After completing these scenarios, the participants were educated and trained in the specifics of the ERC Covid-19 guidelines ${ }^{3-4}$. The training focused on changes of detection of cardiac arrest, covering the face with an oxygen mask with a reservoir and maximum oxygen flow before starting chest compressions, early use of a defibrillator, as well as early videolaryngoscopic intubation with intentional renunciation of chest compressions to minimize aerosol release during intubation ${ }^{1,4}$. If intubation was not possible, participants were able to perform resuscitation using the oxygen mask or bag-mask-ventilation (Covid-19 pandemic-adapted two-helper method). In a special training course, the participants were intensively trained in videolaryngoscopic intubation under medical supervision. 
After this training, the teams were randomized to first complete the scenarios of ERC Covid-19 guidelines with the crew of an ambulance (two paramedics) according to the recommended restriction in staff (Covid-19-minimal-personnel) and then the ERC Covid-19 guidelines in the same team composition as in the control group (Covid-19-advanced-airway-manager) or vice versa.

All teams were given the same scenario in all simulations, which was previously not known to the participants. To enable better comparability, the rhythm in the scenarios was persistent asystole without return-of-spontaneous-circulation. The duration of each scenario was limited to 10 minutes. During the Covid19 scenarios, participants wore personal protective equipment (PPE) as recommended in the Covid-19 guidelines, but importantly, putting on the equipment was not part of the scenarios.

The primary goal was to achieve the highest possible resuscitation quality taking into account the protection specificities due to the Covid-19 pandemic ${ }^{1,3,4}$. The time required for airway management (intervals to application of oxygen, first intubation attempt, successful intubation, duration of intubation attempts, number of intubation attempts, unsuccessful intubation attempts, false intubations) as well as indicators of resuscitation quality according to current ERC guidelines (no-flow-time, total number of compressions, depth of compressions, compression frequency, proportion of compressions with correct handposition and sufficient compression depth, time to first rhythm analysis and to first epinephrine injection, overall dose of epinephrine) were measured ${ }^{3}$.

The Laerdal Resusci Anne QCPR manikin (with legs) (Laerdal Medical GmbH, Lilienthalstr.5, 82178 Puchheim, Germany, http://www.laerdal.com/) was used as manikin. The participants' equipment consisted of a fully equipped emergency backpack, a ventilator (Medumat Standard²; Weinmann Emergency Medical Technology GmbH + Co. KG, Frohbösestraße 12, 22525 Hamburg, Germany, https://www.weinmann-emergency.com) and a transport monitordefibrillator (corpuls3, GS Elektromedizinische Geräte G. Stemple GmbH, Hauswiesenstraße 26, 86916 Kaufering, https://corpuls.world/). For videolaryngoscopic intubation the King Vision ${ }^{\text {TM }}$ aBlade $^{\text {TM }}$ videolaryngoscope from Ambu was used (Ambu GmbH, In der Hub 5, 61231 Bad Nauheim, Germany, https://www.ambu.de).

Data were transmitted via WLAN to the SimPad PLUS (SimPad PLUS, Laerdal Medical 2016, www.laerdal.com) with SkillReporter (Session Viewer, Laerdal Medical 2016, www.laerdal.com), already preinstalled on the tablet. The individual datasets were saved and evaluated using the Debriefing Software Session Viewer 6.2 .6400 by Laerdal. Data were entered into Microsoft Excel Version 2016 for statistical evaluation. SPSS V.20.0 (SPSS V.20.0, IBM, New York, New York, United States of America) was used for statistical analysis.

The primary outcome was no-flow time. Secondary outcomes were the established quality indicators of resuscitation as mentioned above. The differences between the two interventions and control were assessed using a multilevel model to account for repeated measurement obtained on the same team. A sample size of 9 teams was required for a power of $80 \%$ significance level of 0.05 under the assumption that the correlation between the three settings within a team was 0.8 to obtain an effect of 0.33 . In order to test if there are any differences between the two interventions, a correction to the significance level (0.025) was used. Resuscitation data were presented as mean and standard deviation. Differences between intervention groups and control were obtained and tested for significance using random intercept multilevel linear models to adjust for repeated measures by the same teams. A Bonferroni correction was applied to the significance level to allow a further comparison between the two intervention groups.

\section{Results}

Ten different teams of paramedics (male= $80 \%$; female=20\%, average work experience $12 \pm 3$ years) and emergency physicians (male=66\%; female=33\%, average work experience $12 \pm 4$ years) completed a total of 30 resuscitation scenarios apportioned equally to the three study groups. The results of the quality parameters for chest compressions and circulatory interventions are listed in tables 2 and 3.

Table 2: Quality parameters of chest compressions

\begin{tabular}{|c|c|c|c|}
\hline & Control & Covid-19 -minimal-personnel & Covid-19-advanced-airway-manager \\
\hline No-flow-time (sec) & $55 \pm 9$ & $113 \pm 37$ * & $76 \pm 38 \#$ \\
\hline Total number of chest compressions & $1133 \pm 26$ & $985 \pm 78$ * & $1060 \pm 43 \#$ \\
\hline Compression depth (mm) & $57 \pm 2$ & $57 \pm 2$ & $56 \pm 3$ \\
\hline Frequency of compressions (/min) & $126 \pm 2$ & $123 \pm 2$ * & $122 \pm 3$ * \\
\hline \multicolumn{4}{|l|}{ Compressions with } \\
\hline - $\quad$ correct pressure point (\%) & $99 \pm 3$ & $99 \pm 3$ & $97 \pm 5$ \\
\hline - $\quad$ sufficient pressure depth (\%) & $48 \pm 25$ & $52 \pm 21$ & $64 \pm 23$ \\
\hline \multirow[t]{4}{*}{ legend: } & \multicolumn{3}{|c|}{$\mathrm{sec}=$ second; $\mathrm{min}=$ minute; $\mathrm{mm}=$ milimetres } \\
\hline & \multicolumn{3}{|c|}{ * $p<0.001$ vs. Control Group } \\
\hline & \multicolumn{3}{|c|}{$\# p \leq 0.001$ vs. Covid-19 -minimal-personnel } \\
\hline & \multicolumn{3}{|c|}{ data are presented as mean with standard deviation in brackets } \\
\hline
\end{tabular}

Page 4/11 
Table 3: Circulatory interventions

\begin{tabular}{|c|c|c|c|}
\hline & Control & Covid-19 -minimal-personnel & Covid-19-advanced-airway-manager \\
\hline Detection of cardiac arrest (sec) & $16 \pm 4$ & $16 \pm 2$ & $18 \pm 4$ \\
\hline Time to first chest compression (sec) & $21 \pm 5$ & $32 \pm 6$ * & $37 \pm 7$ * \\
\hline Time to first rhythm analysis (sec) & $50 \pm 12$ & $138 \pm 96$ * & $53 \pm 16 \#$ \\
\hline Time to vascular access (sec) & $130 \pm 26$ & $365 \pm 59 *$ & $198 \pm 57 * \#$ \\
\hline Time to first dose of epinephrine (sec) & $161 \pm 39$ & $413 \pm 69$ * & $248 \pm 50 * \#$ \\
\hline Epinephrine total dose applied (mg) & $3.7 \pm 0.7$ & $1.9 \pm 0.6 \pm$ * & $2.9 \pm 0.7 * \#$ \\
\hline legend: & $\begin{array}{l}\text { sec }=\sec 0 \\
\text { * } p<0.001 \\
\# p<0.00 \\
\text { data are }\end{array}$ & $\begin{array}{l}\text { d, mg=miligramm } \\
\text { vs. Control Group } \\
\text { vs. Covid-19- minimal-person } \\
\text { resented as mean with standar }\end{array}$ & deviation in brackets \\
\hline
\end{tabular}

Figure 1 shows the timetable of the different resuscitation interventions and figure 2 the airway and respiratory interventions in the three study groups.

Compared to the control group no-flow-time was prolonged while the total number of chest compressions were reduced in both Covid-19-groups ( $p<0.001$ vs. Covid-19-minimal-personnel)). This could also be observed comparing the Covid-19-groups among themselves ( $p<0.001)$. Quality indicators of chest compressions verified high-quality chest compressions and showed no clinically relevant differences. Nevertheless, a statistical difference in the frequency of chest compressions could be demonstrated ( $p<0.001$ each covid-19-group vs. control group).

Although there was no difference between the three study groups in the time until cardiac arrest was detected, start of chest compressions, first rhythm analysis, establishment of a vascular access and first epinephrine injection occurred later in the Covid-19-minimal-personnel group than in the control group (each $p<0.001$ ). In the Covid-19-advanced-airway-manager group time intervals to start of chest compressions ( $p<0.001$ vs. control group), establishment of a vascular access ( $p<0.001$ vs. control group and vs. Covid-19-minimal-personnel) and first administration of epinephrine ( $<<0.001$ vs. control group and vs. Covid-19-minimal-personnel) were prolonged.

Covering the face and oxygen supply in the Covid-19-groups were faster by passive oxygenation than in the control group by bag-mask-ventilation (each $\mathrm{p}<0.001$ ). Although the first intubation attempt in the Covid-19-groups was earlier than in the control group (each $p<0.001$ ), time to successful intubation did not differ between groups. This was mainly due to the longer intubation time in the Covid-19-minimal-personnel group compared to the Covid-19-advancedairway-manager group $(p=0.002)$ and the control group $(p<0.001)$. Unsuccessful intubation attempts or false intubations were not observed, suggesting a sufficient training.

Comparison of the two Covid-19-groups revealed that the prolonged time intervals to first rhythm analysis, establishment of a vascular access and first epinephrine application were reduced by increasing the number of staff members and the addition of an advanced airway manager ( $p<0.001$ each vs. Covid19-minimal-personnel). While in the control group an average of 3.7 (0.7) mg cumulative epinephrine was applied throughout the scenario, lower cumulative epinephrine doses were applied in Covid-19-minimal-personnel and Covid-19-advanced-airway-manager ( $p<0.001$ each).

\section{Discussion}

The present manikin study examined the effects of the adaptation of the ERC resuscitation guidelines to the Covid-19 pandemic on established quality indicators of resuscitation. Compared to the ERC guidelines of 2015 , no-flow-time was doubled and time intervals to the start of chest compressions, rhythm analysis, vascular access and first dose of epinephrine were prolonged in the recommended minimal team setting. Although oxygen supply and the first intubation attempt were performed earlier, earlier successful intubation was not achieved, because time for airway management was prolonged. Notably, increasing the number of team members and the involvement of an advanced airway manager reduced no-flow-time, time for intubation and time intervals to first rhythm analysis, vascular access and first dose of epinephrine for the Covid-19 algorithm.

In recent years, no-flow-time (interval between collapse and start of chest compression and chest-compression-free time) and low-flow-time (time between start of chest compressions and return of spontaneous circulation) have been identified as central prognostic factors on long-term-survival with good neurological outcome after cardiac arrest ${ }^{10-14}$. Established evidence suggests that even short interruptions in chest compressions worsen patients' outcome $3,10,12,14$. Therefore, the primary focus of efforts to optimize resuscitation has been to minimize no-flow-time, e.g. by starting early, minimizing interruptions and increasing the quality of chest compressions ${ }^{3,10-14}$. The present data indicate that during a 10 min scenario, resuscitation according to the Covid-19 algorithm results in a doubling and therefore significant extension of no-flow-time.Due to a later start as well as a relevant extension of compression-free phases in the context of airway management, which most likely has a negative impact on the neurological outcome. In addition, the interval to first rhythm analysis was markedly prolonged in the Covid-19-minimal-personnel group. This is of potentially high relevance too, because rapid assessment and early

Page 5/11 
defibrillation in case of initial shockable rhythms can significantly improve both, survival rate and neurological outcome in cardiac arrests with shockable rhythms ${ }^{3,10,13}$. Accordingly, the use of a defibrillator is recommended early in the Covid-19 and ERC-2015 algorithms, assuming that it does not lead to increased aerosol release but significantly improves the outcome of the affected patients ${ }^{3,4}$. Potential reasons for the present results could be an increased focus on airway coverage and preparation/performance of intubation as well as the reduced number of staff members. The later hypothesis is supported by the significant attenuation of the delay in first rhythm analysis in the Covid-19-advanced-airway-manager group. However, in the later setting there is an increased number of staff members at risk of infection. Through improved training and increased awareness, it might be possible to improve the time to the first rhythm analysis even with a reduced number of providers.

Taken together, the protection of the resuscitation providers must be balanced against the potential impairment of outcome for the patients. In view of the large locoregional variability of Covid-19 between and within the affected countries ${ }^{4}$, applying the Covid-19 algorithm in general would potentially expose Covid-19-negative patients to the risk of a worse outcome. Facing the current pandemic situation, it appears necessary to define specific indicators to differentiate between the performance of two resuscitation guidelines with respect to patient care and the safety of the medical personnel. In addition to evidence or symptoms of Covid-19, the contact status of the patient and the daily incidence of Covid-19 on site are of particular importance ${ }^{15}$. Professional resuscitation providers are therefore faced with special challenges to guarantee optimal care for the patients concerned: in addition to the consistent use of appropriate personal protective equipment associated with specific risks of lowering resuscitation quality (e.g. early fatigue, deterioration of intubation and access conditions), they not only need to be trained in both resuscitation algorithms as well as in airway management, but also in decision pathways to choose the appropriate algorithm.

The implementation of optimal airway management during resuscitation has been the aim of various large-scale studies in recent years and has been a subject of controversial discussion.

While studies within ACLS for professional resuscitation assistants emphasized the importance of bag-mask-ventilation ${ }^{3,16,17}$, Wang and co-workers were able to demonstrate a significant improvement in the 72-hour-survival-rate for initial airway protection using a laryngeal tube in adult patients with out-ofhospital cardiac arrest ${ }^{18}$. Benger and co-workers., on the other hand, could not demonstrate a favorable effect on the functional outcome in the Airways-2study for the initial use of a supraglottic airway device compared to endotracheal intubation after 30 days ${ }^{19}$. These data support that the importance of advanced airway management was considered secondary to the implementation of early defibrillation and minimal no- or low-flow-time in the 2015 ERC guidelines ${ }^{3}$. In view of the transmission path of the SARS-CoV-2 pathogen, Covid-19 guidelines focus on minimization of aerosol release to protect the providers from infection ${ }^{1,4,5}$. Based on the assumption that a sufficiently blocked endotracheal tube using a suitable virus filter can effectively prevent aerosol release and thus reduce the exposure of providers, early endotracheal intubation is recommended in Covid-19 guidelines with the interruption of chest compressions, for which there are indications in recent manikin studies $1,4,5,20$. Since the use of videolaryngoscopy increases the first-pass-success rate even for inexperienced assistants and also allows the airway-manager a greater distance to the mouth, nose and throat, it is currently explicitly recommended 4, 21. In the present study, an earlier start of the first intubation attempt could be observed for resuscitation according to the Covid-19 algorithm, but the time until successful intubation did not differ between the three groups despite the use of a videolaryngoscope. Notably, specifically in the Covid-19-minimalpersonnel group there was a significant extension of the intubation time and thus a relevant extension of no-flow-time. Deterioration of intubation conditions could additionally be caused by using eye and face protection. However, both Covid-19 groups wore PPE. Although the paramedics in Covid-19-minimalpersonnel had intensive training in performing videolaryngoscopic intubation before the scenarios were carried out, there was a high variability of intubation duration in the Covid-19-minimal-personnel group. While this observation might be reduced by regular training in the future, given the dynamics of the current pandemic situation, it seems unrealistic to train every preclinical paramedic sufficiently in the technique of videolaryngoscopic intubation and to equip every rescue vehicle with a videolaryngoscope at short-term.

The present data are in agreement with previous study results showing that prolonged intubation attempts are associated with an increased no-flow-time, which may lead to a delay of return of spontanoues circulation and worsen the outcome ${ }^{3,17}$. Therefore, the presence of an experienced airway manager in these emergency situations may be beneficial. This assumption is supported by the results of the Covid-19-advanced-airway-manager group, that revealed a marked reduction of the time interval to successful intubation as well as the duration of intubation. These results might foster a discussion about the heterogeneity of pre-clinical systems in various countries with the existence of non-emergency physician-based as well as inter-professional character of emergency physician-based systems such as in Germany against the background of the current Covid-19 pandemic ${ }^{22}$.

While covering the face with an aid (e.g. a jacket or similar) is recommended for the lay helpers, covering the airways with an oxygen mask with reservoir is suitable and recommended in connection with professional emergency care in the Covid-19 algorithm ${ }^{4}$. Using an oxygen mask allows the covering of the mouth, nose and throat area as well as an initial passive oxygenation. In a recently published manikin study, which explored strategies to reduce aerosolspread during chest compressions, an oxygen mask on the patient's face deflected the spread. Therefore it might be suitable to reduce the exposure of providers ${ }^{20}$. In studies of passive oxygenation, an improvement in the survival rate and neurological outcome was demonstrated for the first minutes of resuscitation even without airway intervention ${ }^{23-25}$. The present study shows that passive oxygenation using the Covid-19 algorithm can lead to an earlier application of oxygen than in the previous ERC 2015 algorithm. Therefore, this could represent a reasonable compromise between necessary provider protection on the one hand with a deflection of aerosol-spread and optimal patient care on the other hand, ensuring early oxygen application for the patients, while bag-mask-ventilation or airway interventions are prepared $20,23-25$

The current study has some limitations that need to be acknowledged. The installation of personal protective equipment was not included in the individual time intervals. Therefore, a further extension of no-flow-time and additional time intervals might be possible as observed in studies of SARS ${ }^{26,27}$. The scenarios consisted only of non-shockable rhythms. However, it is unlikely that the no-flow time in a corresponding scenario with a shockable rhythm shall

Page 6/11 
deviate similar. Performing resuscitation under investigation during the study may have influenced the participants and thus may have altered the results (Hawthorne effect). Furthermore, manikin-based investigations provide only a limited reproduction of reality and patients'outcome could not be evaluated. However, the effects are highly significant and in view of the current situation with the spread of the Covid-19 pandemic and the expected deterioration in the quality of resuscitation, there are only limited possibilities for the urgently required clinical evaluation of the corresponding guideline changes. In addition, results for the Covid-19 guidelines may improve following more intensified training of the providers. On the one hand training success was high, as

suggested by the lack of false intubations, for example. On the other hand, due to the pandemic even training of the medical staff is very difficult to organize.

\section{Conclusions}

Our manikin study showed that COVID-19 guidelines for a non-shockable cardiac arrest led to earlier attempts at intubation, delay in starting chest compressions and longer interruption in chest compression, which markedly worsen the quality of cardiopulmonary resuscitation. These negative effects are attenuated by increasing the number of team members and the involvement of an experienced airway manager. Specific indications for the Covid-19 ERC guidelines are urgently required to carefully balance the risk of infection with Sars-CoV-2 for providers vs. the potentially worse outcome for the patients.

\section{List Of Abbreviations}

ACLS Advanced-Cardiac-Life-Support

BLS Basic-Life-Support

Covid-19 Corona-Virus-Disease 2019

CPR Cardio-pulmonary Resuscitation

e.g. exempli gratia

ERC European Resuscitation Council

HEPA High Efficiency Particulate Air filter

HME Heat and moisture exchanger filter;

PPE Personal protective equipment

SARS Severe acute respiratory syndrome

SARS-CoV-2 Severe acute respiratory syndrome coronavirus type 2

Sec Seconds

WLAN Wireless Local Area Network

\section{Declarations}

\section{Ethics approval and consent to participate}

Ethical approval for this study was provided by the Ethical Committee of the University Hospital of Muenster, Muenster, Germany (Ethical Committee No 2020-504-f-S). All participitants gave informed consent

\section{Consent for publication}

Not applicable

\section{Availability of data and materials}

The datasets used and/or analysed during the current study are available from the corresponding author on reasonable request.

\section{Competing interests}

Gerrit Jansen, Nicole Ebeling, Eugen Latka, Stefan Krüger, Sean Scholz, Simon Trapp, Julia Johanna Grannemann, Daniel Thaemel, Suraj Chandwani, Odile Sauzet and Rainer Borgstedt not indicate a conflict of interest. Sebastian Rehberg SR. is a medical advisor for Fresenius Kabi Germany, has received honoraria and travel expenses from Amomed Pharma and Orion Pharma.

\section{Funding}

This work was solely supported by institutional and departmental sources. 
GJ: Study design, Patient recruitment, data collection, data analysis and writing up of the first draft of the paper

NE: substantial contribution to conception and design, acquisition of data, or analysis and interpretation of data

EL: Patient recruitment, data collection, data analysis, interpretation of data and final approval

SK: Patient recruitment, data collection, data analysis, interpretation of data and final approval

SS: Patient recruitment, data collection, data analysis, interpretation of data and final approval

ST: Patient recruitment, data collection, data analysis, interpretation of data and final approval

JJG: Patient recruitment, data collection, data analysis, interpretation of data and final approval

DT: Patient recruitment, data collection, data analysis, interpretation of data and final approval

SC: Patient recruitment, data collection, data analysis, interpretation of data and final approval

OS: Data collection, data analysis, interpretation of data and final approval

RB: data analysis, interpretation of data and final approval

SR: Study design, Patient recruitment, data collection, data analysis and final approval

\section{Acknowledgements}

We would like to thank Mrs. Franziska Behrens and Mr. Andreas Stenzel for the graphics.

\section{References}

[1] Gräsner J-T, Bohn A, Seewald S, Bein B, Fischer M, Wnent J. COVID-19 und Herz-Kreislauf-Stillstand: Aktuelle Anpassung der Guidelines 2015. Anästh Intensivmed. 2020;61:148-53.

[2] German Resuscitation Council (GRC) Statement on the implementation of resuscitation measures in the context of the COVID-19 pandemic 2020. 2020. https://www.grcorg.de/files/ArticleFiles/document/Stellungnahme\%20des\%20Deutschen\%20Rats\%20fur\%20Wiederbelebung\%20zu\%20CPR\%20bei\%20Corona\%202020.pdf Accessed 16 Jul 2020).

[3] Soar J, Nolan JP, Böttiger BW, Perkins GD, Lott C, Carli P et al. European Resuscitation Council Guidelines for Resuscitation 2015: Section 3. Adult advanced life support. Resuscitation. 2015;95:100-47.

[4] Olasveengen T, Castrén M, Handley A et al. Basismaßnahmen zur Wiederbelebung Erwachsener: COVID-19-Leitlinien des European Resuscitation Council. Notf Rett Med. 2020;23:246-7.

[5] Couper K, Taylor-Phillips S, Grove A et al. COVID-19 in cardiac arrest and infection risk to rescuers: A systematic review. Resuscitation. 2020;151:59-66.

[6] Loeb M, McGeer A, Henry B et al. SARS among critical care nurses. Emerging Infect Dis. 2004.;10:251-5.

[7] Raboud J, Shigayeva A, McGeer A et al. Risk factors for SARS transmission from patients requiring intubation: A multicentre investigation in Toronto, Canada. PLoS ONE. 2010;5:e10717.

[8] Liu W, Tang F, Fang L-Q et al. Risk factors for SARS infection among hospital healthcare workers in Beijing: A case control study. Tropical Medicine \& International Health. 2009;14:52-9.

[9] Perkins GD, Morley PT, Nolan JP et al. International Liaison Committee on Resuscitation: COVID-19 consensus on science, treatment recommendations and task force insights. Resuscitation. 2020;151:145-7.

[10] Adnet F, Triba MN, Borron SW et al. Cardiopulmonary resuscitation duration and survival in out-of-hospital cardiac arrest patients. Resuscitation. 2017;111:74-81.

[11] Hasselqvist-Ax I, Riva G, Herlitz J et al. Early cardiopulmonary resuscitation in out-of-hospital cardiac arrest. N Engl J Med. 2015;372:2307-15.

[12] Cha WC, Lee EJ, Hwang S-S. The duration of cardiopulmonary resuscitation in emergency departments after out-of-hospital cardiac arrest is associated with the outcome: A nationwide observational study. Resuscitation. 2015;96:323-7.

[13] Grunau B, Reynolds JC, Scheuermeyer FX et al. Comparing the prognosis of those with initial shockable and non-shockable rhythms with increasing durations of CPR: Informing minimum durations of resuscitation. Resuscitation. 2016;101:50-6. 
[14] Goldberger ZD, Chan PS, Berg RA et al. Duration of resuscitation efforts and survival after in-hospital cardiac arrest: An observational study. Lancet. 2012;380:1473-81.

[15] Zheng Z, Peng F, Xu B et al. Risk factors of critical \& mortal COVID-19 cases: A systematic literature review and meta-analysis. J Infect. 2020;81:e16e25.

[16] Hasegawa K, Hiraide A, Chang Y, Brown DFM. Association of prehospital advanced airway management with neurologic outcome and survival in patients with out-of-hospital cardiac arrest. JAMA. 2013;309:257-66.

[17] Bernhard M, Benger JR. Airway management during cardiopulmonary resuscitation. Curr Opin Crit Care. 2015;21:183-7.

[18] Wang HE, Schmicker RH, Daya MR et al. Effect of a Strategy of Initial Laryngeal Tube Insertion vs Endotracheal Intubation on 72-Hour Survival in Adults With Out-of-Hospital Cardiac Arrest: A Randomized Clinical Trial. JAMA. 2018;320:769-78.

[19] Benger JR, Kirby K, Black S et al. Effect of a Strategy of a Supraglottic Airway Device vs Tracheal Intubation During Out-of-Hospital Cardiac Arrest on Functional Outcome: The AIRWAYS-2 Randomized Clinical Trial. JAMA. 2018;320:779-91.

[20] Ott M, Milazzo A, Liebau S, et al. Exploration of strategies to reduce aerosol-spread during chest compressions: A simulation and cadaver model. Resuscitation. 2020;152:192-8.

[21] Baek MS, Han M, Huh JW, Lim C-M, Koh Y, Hong S-B. Video laryngoscopy versus direct laryngoscopy for first-attempt tracheal intubation in the general ward. Ann Intensive Care. 2018;8:83.

[22] Chenaitia H, Massa H, Noel C et al. Paramedics in prehospital emergency medical systems across Europe. International Paramedic Practice. 2011;1:339.

[23] Ewy GA. A new approach for out-of-hospital CPR: A bold step forward. Resuscitation. 2003;58:271-2.

[24] Bobrow BJ, Ewy GA, Clark L et al. Passive oxygen insufflation is superior to bag-valve-mask ventilation for witnessed ventricular fibrillation out-ofhospital cardiac arrest. Ann Emerg Med. 2009;54:656-62.

[25] Deakin CD, O'Neill JF, Tabor T. Does compression-only cardiopulmonary resuscitation generate adequate passive ventilation during cardiac arrest? Resuscitation. 2007;75:53-9.

[26] Abrahamson SD, Canzian S, Brunet F. Using simulation for training and to change protocol during the outbreak of severe acute respiratory syndrome. Crit Care. 2006; doi:10.1186/ cc3916.

[27] Watson L, Sault W, Gwyn R, et al: The "delay effect" of donning a gown during cardiopulmonary resuscitation in a simulation model. CJEM. 2008; doi:10.1017/s1481803500010332.

\section{Figures}




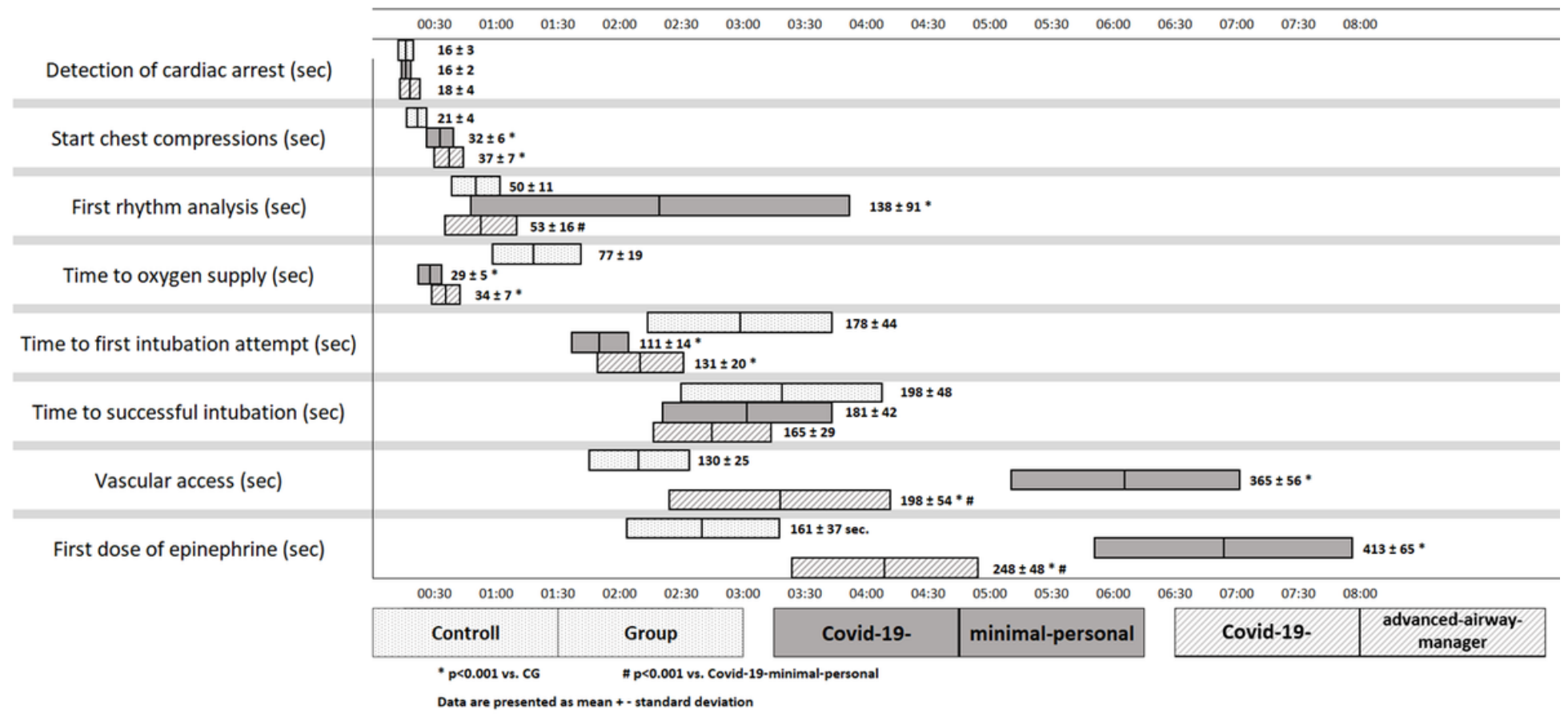

\section{Figure 1}

Timetable of resuscitation interventions 

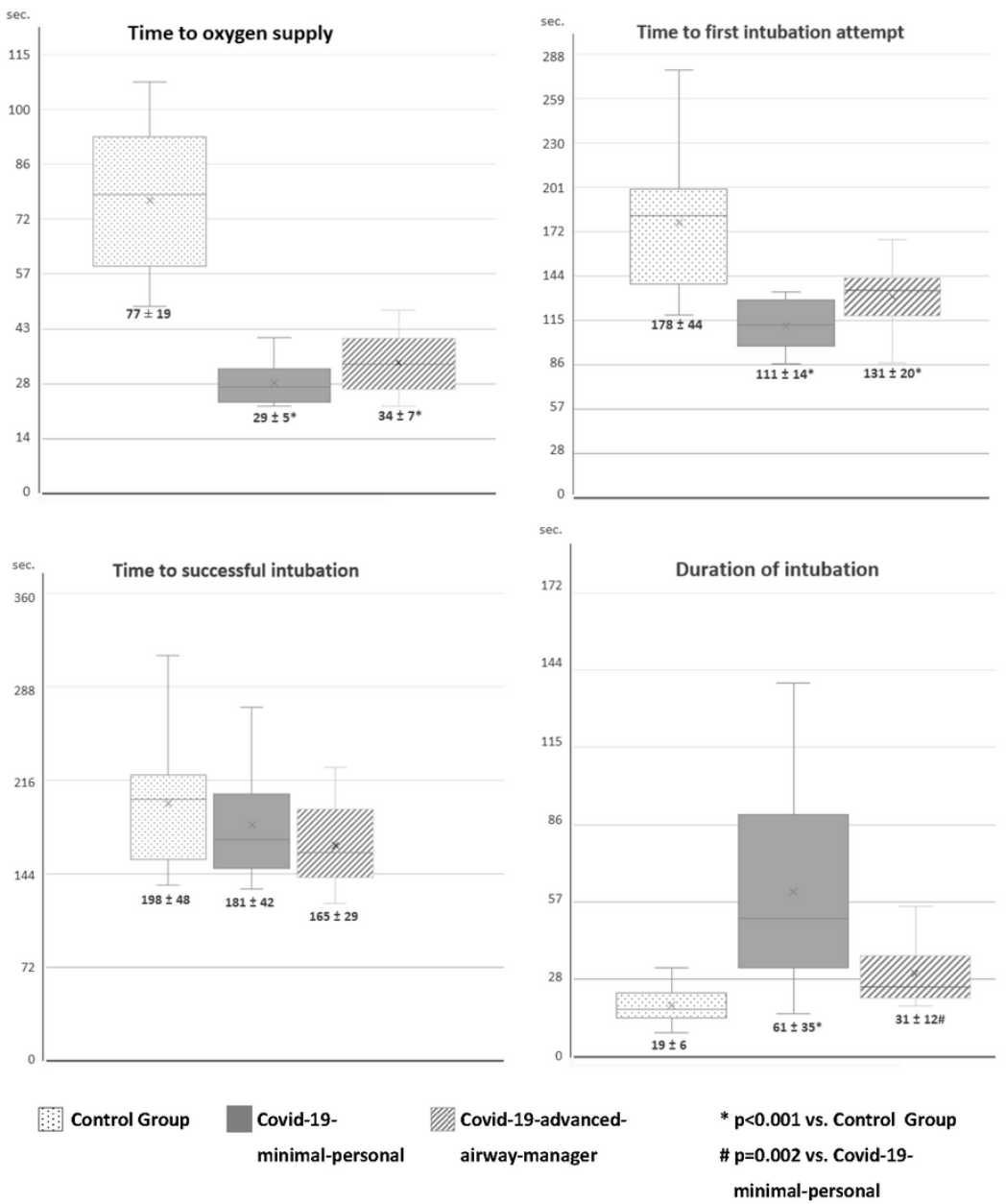

Figure 2

Airway and respiratory interventions 